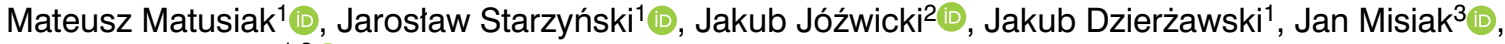
Wojciech Jóźwicki ${ }^{1,3}$ (iD

${ }^{1}$ Department of Tumour Pathology and Pathomorphology, Oncology Centre-Prof. Franciszek Łukaszczyk Memorial Hospital, Bydgoszcz 85-796, Poland

2Department of Clinical Pathomorphology, Faculty of Medicine, Ludwik Rydygier Collegium Medicum in Bydgoszcz, Nicolaus Copernicus University in Torun, Bydgoszcz, Poland

32Department of Tumour Pathology and Pathomorphology, Nicolaus Copernicus University Collegium Medicum in Bydgoszcz, Bydgoszcz, Poland Mateusz Matusiak and Jarosław Starzyński are equal first authors.

\title{
The type of distribution of PD-L1 positive immune cells and PD-L1 expression in tumor cells correlate with the development of non-classical differentiation in urinary bladder cancer
}

\author{
Corresponding author: \\ Wojciech Jóźwicki, Department \\ of Tumour Pathology \\ and Pathomorphology, \\ Oncology Centre-Prof. \\ Franciszek Łukaszczyk Memorial \\ Hospital, Bydgoszcz 85-796, \\ Romanowskiej 2 St., Poland, \\ e-mail: jozwickiw@co.bydgoszcz.pl
}

Medical Research Journal 2019; Volume 4, Number 3, 136-141 10.5603/MRJ.a2019.0025 Copyright (C) 2019 Via Medica ISSN 2451-2591

\begin{abstract}
Background: The basic diagnostic tool of urinary bladder cancer is the histopathological assessment. However, it is insufficient to accurately predict the progression of this disease. There is a need to look for new prognostic factors that will make the therapeutic process more effective. The aim of this study is to evaluate the effect of activation of a PD1 - PD-L1 immune checkpoint in immune effector cells (IECs) and tumor cells, on the development of malignancy in the form of non-classic differentiation in urinary bladder cancer.

Materials and methods: 110 patients with stage pT1-pT4 urothelial bladder carcinoma who underwent radical cystectomy/cystoprostatectomy between 2011 and 2014 were included in the study. Tumor advancement (pT stage), grade (G), as well as, non-classic differentiation frequency and number were evaluated pathologically. In each case, the area of the tumor containing PD-L1+ IECs was analyzed. The distribution of PD-L1 + immune effector cells within the tumor was also assessed as dispersed or aggregated.

Results: The frequency of non-classic differentiation was significantly lower in urothelial bladder cancer tumors with a dispersed pattern of distribution of PD-L1 + IECs. A correlation between the extent of PD-L1 expression in tumor cells and the non-classic differentiation number in UBC was identified.

Conclusions: The distribution of cells expressing the immune checkpoint biomarker PD-L1 constitutes a new prognostic factor and may play a key role in the selection of individualized immunotherapy. In addition, the evaluation of non-classic differentiation in the tumor may complement the assessment of PD-L1 expression due to its capacity to characterize the current malignant potential of the tumor, whereas the assessment of extent and distribution of PD-L1 + in tumor-associated immune cells indicates the functional status of the immune system.

Key words: PD-L1; urothelial bladder cancer; tumor microenvironment; immune cell, NDN, immune effector cells, IEC, immunological control point distribution, ICPD
\end{abstract}

Med Res J 2019; 4 (3): 136-141

\section{Introduction}

Histopathological assessment is an important tool in the diagnostics of urinary bladder cancer (UBC). The diagnostic standard of UBC includes the evaluation of the histological type and advancement defined by the depth of invasion (pT), lymph node status (pN), as well as the estimation of histological malignancy (G). These features are commonly acknowledged as prognostic factors [1]. However, accurate prediction of the UBC progression is still not possible. In this context, there is an urgent need to look for factors that 
better characterize the development of the malignant potential of urinary bladder cancer. Literature sources have demonstrated that cases with non-classic differentiation (ND) such as nested, micropapillary, sarcomatoid or lymphoepithelial-like differentiation have a poorer prognosis. The Classification of Malignant Tumours of the Urinary System issued by the WHO in 2004 , included as many as 12 types of ND that could develop in urinary bladder cancer (squamous, glandular, trophoblastic, nested, microcystic, micropapillary, lymphoepithelial-like, lymphoma-like, plasmacytoid, sarcomatoid, and giant cell differentiation, as well as the non-differentiated type), confirming the significant value of including it in routine diagnostics [2]. Since then, a new approach and methodology have been developed for assessing ND as a prognostic factor in UBC. It was demonstrated that a higher percentage of ND and the higher number of different non-classical types of differentiation in one tumor was associated with shorter survival time in patients with $\operatorname{UBC}[3,4]$. The development of ND in UBC is accompanied by various molecular phenomena, such as the expression of RCAS1, OCT4 or the increased frequency of regulatory $T$ cells [5-7]. Recently, researchers have been interested in the issue of immune checkpoints (ICP) that influence the efficiency of effector cells of the immune system [8, 9]. One of them is the PD1-PD-L1 signalling pathway. Its activation in the neoplastic process is associated with a significant reduction or even complete elimination of the anti-cancer immune system response $[9,10]$. The aim of this study is to evaluate the effect of activation of PD1 - PD-L1 immune control point in immune effector cells (IECs) and tumor cells (TCs), on the development of malignancy, in the form of ND in urinary bladder cancer.

\section{Materials and methods}

110 patients with pT1-pT4 urothelial bladder cancer who underwent radical cystectomy/cystoprostatectomy between 2011 and 2014 were included in the study. The experimental group was comprised of patients treated at Oncology Centre Prof. Franciszek Łukaszczyk Memorial Hospital in Bydgoszcz (Poland). The mean age of patients was 65 years (65 years in women and in men). The clinico-pathomorphological characteristics of the tumors in the study group are presented in Table 1.

The staging and histological classification of tumors were assessed according to the WHO Classification of Tumors. The study was approved by the Committee of Ethics of Scientific Research of Collegium Medicum, Nicolaus Copernicus University, Poland (KB 587/2018).
Table 1. Patient clinico-pathological characteristics

\begin{tabular}{lc}
\hline Patient's characteristics & Number of patients \\
\hline Sex & 22 \\
Female & 88 \\
Male & \\
pT & 12 \\
1 & 13 \\
$2 a$ & 12 \\
$2 b$ & 10 \\
$3 a$ & 31 \\
$3 b$ & 32 \\
$4 a$ & 0 \\
$4 b$ & \\
G & 1 \\
no available & 2 \\
1 & 47 \\
2 & 60 \\
3 & \\
NDN & 34 \\
0 & 26 \\
1 & 25 \\
2 & 25 \\
$\geq 3$ &
\end{tabular}

\section{The preparation and assessment of H\&E stained samples}

Tissue sections were fixed in $10 \%$ buffered formalin and embedded in paraffin blocks according to a standard protocol. Tumor advancement (pT stage), histological malignancy $(G)$, frequency of ND and the number of non-classic differentiation types (NDN) was assessed as previously described $[3,11]$.

\section{The preparation and assessment of immunostained samples}

Paraffin blocks most representative of the tumor were selected for immunohistochemistry staining. $4 \mu \mathrm{m}$ sections were stained with rabbit anti-PD-L1 (Ventana clone SP142, Roche) and evaluated with OptiView DAB IHC Detection Kit and OptiView Amplification Kit using the Ventana BenchMark system according to the manufacturer's protocol. To confirm the specificity of the signal, for each sample a negative control stained without anti-PD-L1 antibody was provided. For each staining cycle, a positive control sample of human tonsil was included following the manufacturer's recommendations. In each test sample, the percent of tumor area occupied by tumor-associated immune cells exhibiting PD-L1 positive staining was assessed. IECs that were assessed included lymphocytes, macrophages, den- 

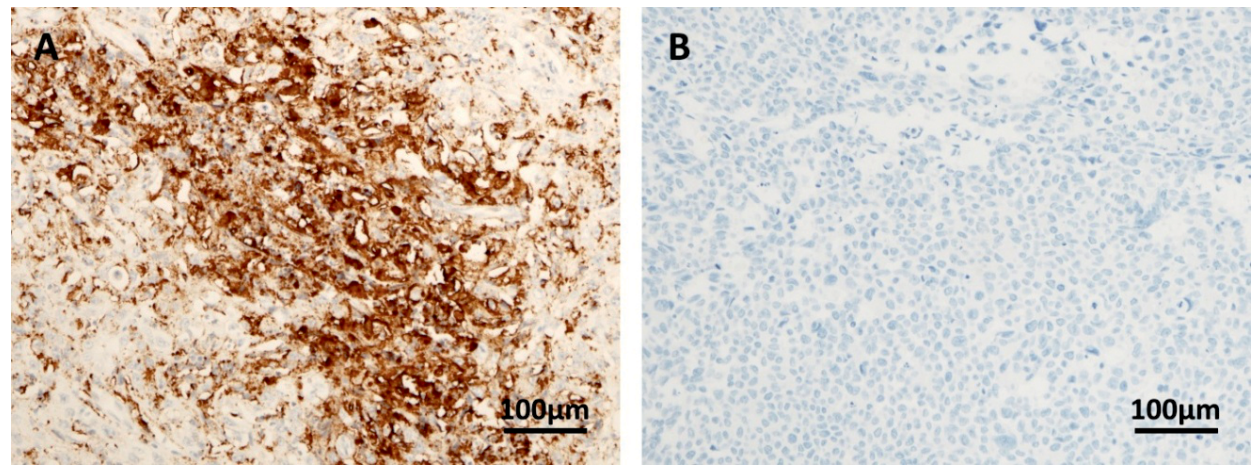

Figure 1. The sample images show the presence of PD-L1 expression in tumor cells $(A)$ and its absence $(B)$
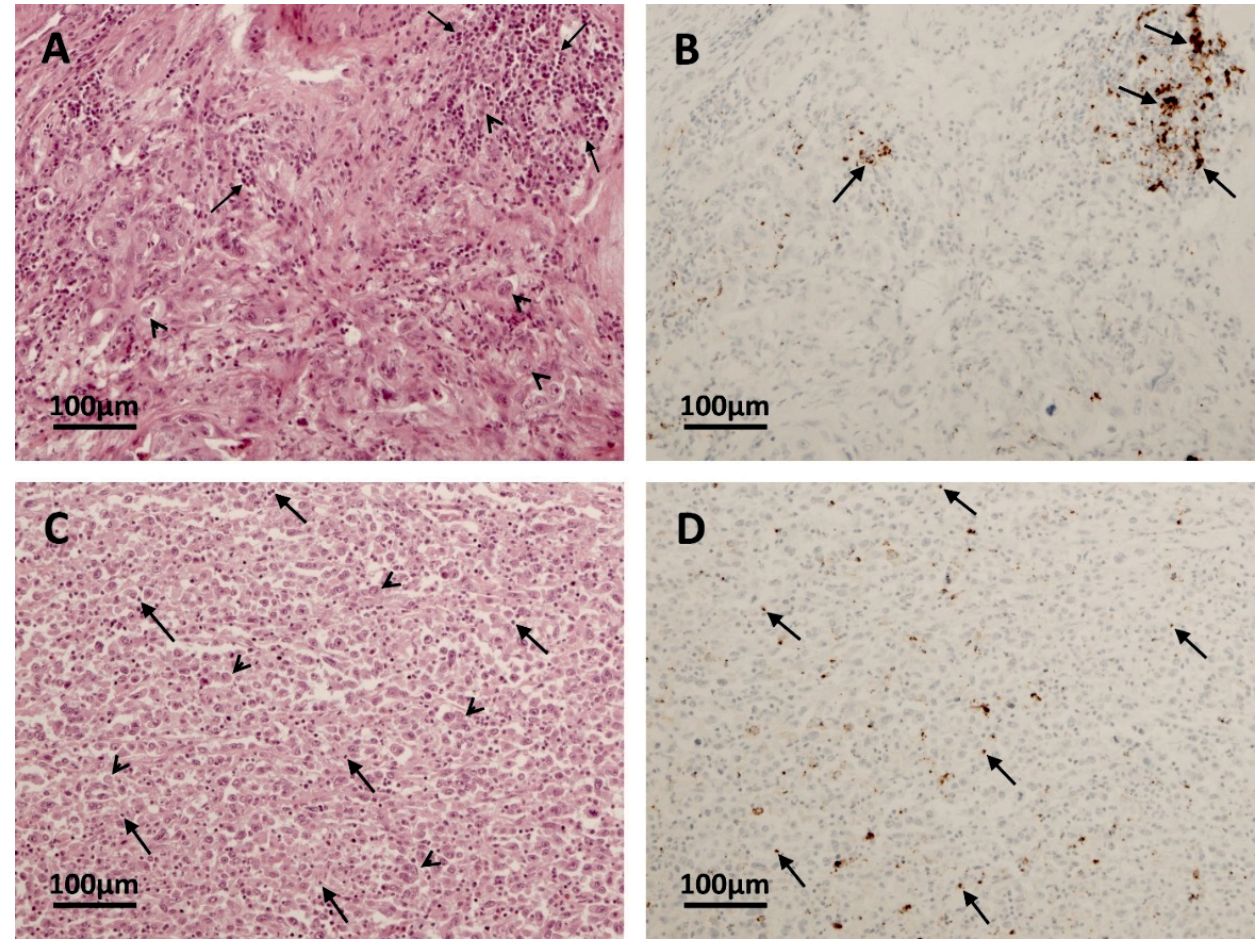

Figure 2. A low percentage of non-classic differentiation in the tumor $(A)$ (case with $N D=10 \%$ ) correlates with a dispersed distribution pattern of PD-L1 + IECs (B). An aggregated distribution pattern of PD-L1 IECs (D) correlates with a high percentage of non-classic differentiation in the tumor $(C)$ (case with ND=85\%). Arrows indicate PD-L1 + IECs, arrowheads indicate tumor cells

dritic cells and granulocytes. The pattern of distribution of PD-L1 + IECs within the tumor was determined as either dispersed (Fig. 2D) or aggregated (Fig. 2B) [12]. In tumor cells, the expression of PD-L1 was assessed as either positive (Fig. 1A) or negative (Fig. 1B).

\section{Statistical analysis}

The relationships between expression of PD-L1 on IECs and TCs and variables such as $\mathrm{PT}, \mathrm{G}$, the extent of non-classic differentiation and NDN were analyzed using T-test for independent samples. The statistical analyses were performed using STATISTICA data analysis software (version 8.0; StatSoft, Inc., Tulsa,
OK, USA). A p-value $<0.05$ was considered to be significant.

\section{Results}

PD-L1 positive immune cells and non-classic differentiation

A correlation between the extent of non-classic differentiation and the distribution pattern of IECs expressing PD-L1 in UBC was observed (Fig. 2). ND frequency was significantly lower in tumors with a dispersed distribution pattern of PD-L1 + IECs (Fig. 3). 


\section{PD-L1 expression in tumor cells and NDN}

The expression of PD-L1 on tumor cells was correlated with NDN in UBC samples (Fig. 4). In tumors

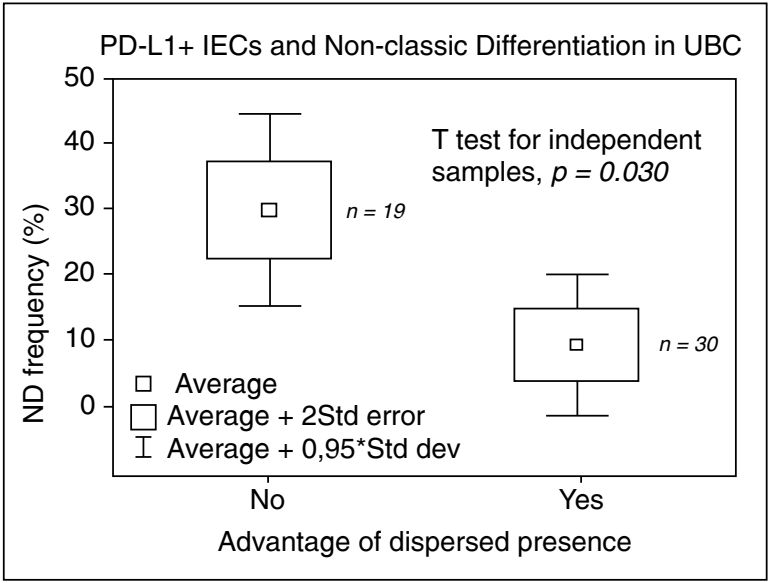

Figure 3. In tumors presenting high dynamics of invasion, in which the expression of PD-L1 includes at least $80 \%$ of tumor cells $(n=49)$, the extent of non-classical differentiation in tumors with the advantage of the aggregated presence of PD-L1 + IECs was almost twice as much as in tumors with a predominance of dispersed PD-L1 + IECs presence with the presence of non-classic differentiation types (NDN >0), the extent of PD-L1 expression in tumor cells was significantly higher compared to tumors without non-classic differentiation type (NDN = 0) (Fig. 5).

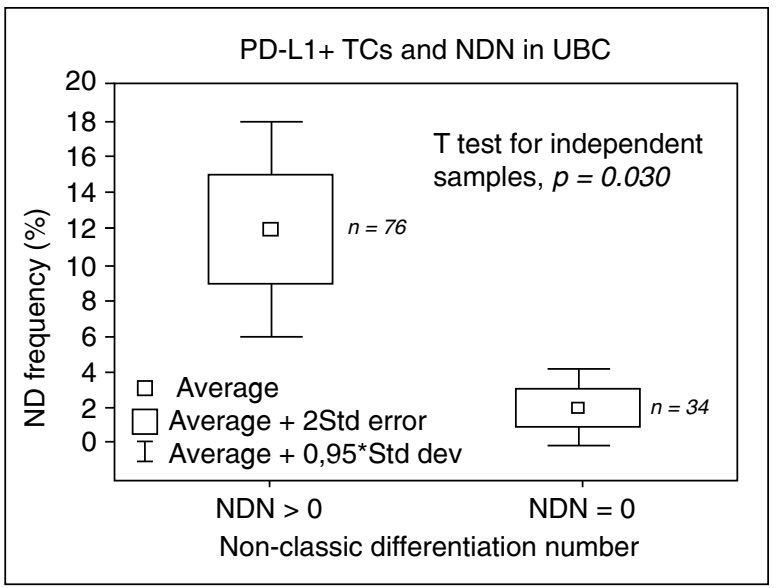

Figure 5. The extent of PD-L1 expression in tumor cells was at least six times higher in tumors with non-classical differentiations
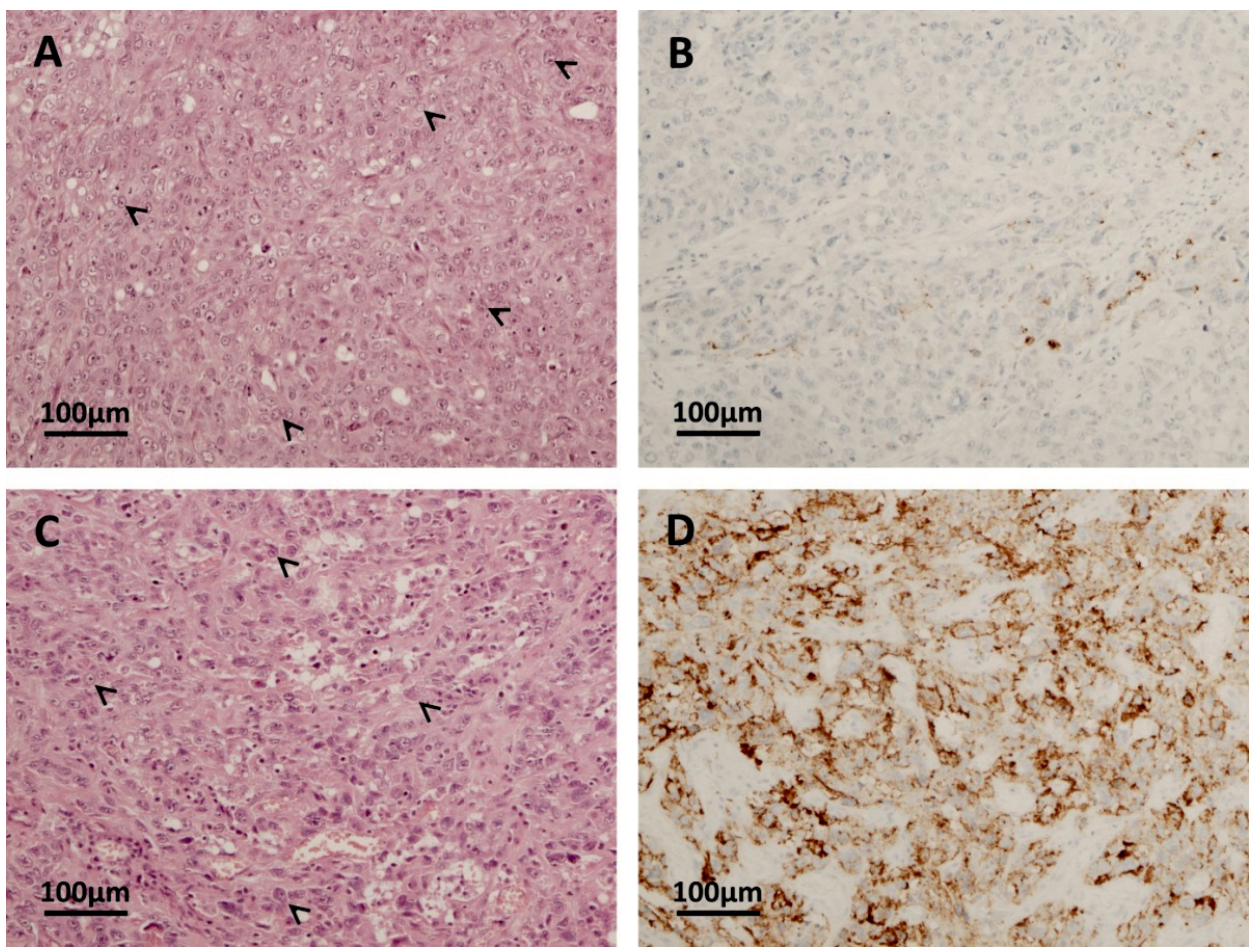

Figure 4. A low number of non-classic differentiations $(A)$ is associated with a low frequency of tumor cells expressing PD-L1 (B) (case with NDN = 0 and \%TCs-PD-L1 + = 1\%). A higher frequency of PD-L1+ tumor cells (D) can be observed in tumors with a higher number of non-classic differentiation types (C) (case with NDN $=2$ and \%TCs-PD-L1 $+=80 \%$ ) 
PD-L1 expression in neoplastic cells and tumor stage $(\mathrm{pT})$

There was no significant correlation between PDL1 frequency and tumor staging (not shown).

\section{PD-L1 expression in neoplastic cells and tumor grade $(\mathrm{G})$}

There was no significant correlation between PDL1 frequency and tumor grading (not shown).

\section{Discussion}

Evaluation of PD-L1 expression in cells within a tumor area is a multifaceted source of information on the developmental status of a tumor. Activation of the PD-1 - PD-L1 signalling pathway may result in not only a reduction of anti-cancer immune system response [9, 10] but also drive tumor progression and malignancy as evidenced by increased non-classic differentiation in UBC [13]. Our results suggest that the effectiveness of the PD1 - PD-L1 immune checkpoint might depend not only on the expression of the ligand protein PD-L1 on IECs but also the pattern of distribution of those cells within the tumor. A dispersion of PD-L1 positive immune cells does not favour the frequency of non-classic differentiation in the tumor (Fig. 2A, B). Conversely, ND is significantly higher in tumors with an aggregated pattern of distribution of PD-L1+ IECs (Fig. 2C, D, Fig. 3). This pattern of expression is consistent with the proposed mechanism of immune checkpoints and may be of key importance in planning immunotherapy strategies as the upregulation of PD-L1 expression on tumor cells is often accompanied by an increased expression on IECs as well [14]. Suppression of IECs activity by the activation of immune checkpoint signalling pathways may promote the progression of UBC $[9,15]$. According to our knowledge, the pattern of distribution of PD-L1+ cells has not been evaluated in UBC before. The second parameter describing non-classic differentiation we assessed was NDN which is independent of the extent of ND in the tumor [3]. In our study, we observed an increase in NDN within tumors with a higher frequency of PD-L1 positive TCs (Fig. 4, Fig. 5). An increase in the number of non-classic histological types of differentiation underlies an increase in tumor malignancy even if the extent of ND as a whole constitutes only a small percentage of the tumor area $[3,4,11]$. Pichler et al. demonstrated that the assessment of PD-L1 expression on IECs is the most reliable prognostic marker of tumor response to therapy. That study also highlighted high heterogeneity of PD-L1 expression which may help explain divergent results [16]. Altogether, an assessment of activated immune checkpoint pathways, expression of PD-L1 as well as the distribution of PD-L1 positive cells within the tumor should be a part of a standard evaluation of tumor immunological status in urothelial bladder cancer. In our study, the expression of PDL1 and distribution of PD-L1 + cells was not correlated with tumor advancement (pT) and grade $(G)$. There is no consensus on that matter in literature. In a study on lung adenocarcinoma, a correlation between high histological differentiation and PD-L1 expression on TCs and IECs was found [17]. Jabbour et al. found a correlation between PD-L1 expression on TCs and IECs and tumor advancement [18]. However, other studies did not confirm these findings [19] in line with our own results. Numerous studies confirm the efficacy of inhibitors of control points in cancer immunotherapy [20-26]. However, not all patients respond to anti-PD-L1 therapy to the same extent which highlights the need to search for novel biomarkers to further stratify the treatment groups $[27,28]$.

\section{Conclusions}

The pattern of expression of immune checkpoint biomarkers such as PD-L1 in tumors is a new predictive tool and a potential prognostic factor in patients with UBC. An assessment of dispersion or aggregation patterns of cells expressing immune checkpoint biomarkers may play a major role in tailoring individualized immunotherapy. Moreover, the evaluation of $\mathrm{ND}$ in the tumor may complement the assessment of PD-L1 expression due to its capacity to characterize the current malignant potential of the tumor, whereas the assessment of extent and distribution of PD-L1 + in tumor-associated immune cells indicates the functional status of the immune system.

\section{Grant support}

This study was supported in part by funds for statutory research from Collegium Medicum, Nicolaus Copernicus University.

\section{Conflicts of interest}

The authors declare no conflicts of interest.

\section{List of abbreviations}

$$
\begin{aligned}
& \text { IEC - immune effector cells } \\
& \text { TC - tumour cells } \\
& \text { PD-L1 - programmed death ligand 1 } \\
& \text { PD-1 - programmed death 1 } \\
& \text { ICI - immune checkpoint inhibitors } \\
& \text { UBC - urothelial bladder cancer }
\end{aligned}
$$




\section{References}

1. Bellmunt J, Orsola A, Leow JJ, et al. ESMO Guidelines Working Group. Bladder cancer: ESMO Practice Guidelines for diagnosis, treatment and follow-up. Ann Oncol. 2014; 25 Suppl 3: iii40-iii48, doi: 10.1093/annonc/mdu223, indexed in Pubmed: 25096609.

2. Crundwell M. Pathology and genetics of tumours of the urinary system and male genital organs. BJU International. 2004; 94(4): 675-675, doi: 10.1111/j.1464-410x.2003.05068.x.

3. Jozwicki W, Domaniewski J, Skok Z, et al. Usefulness of histologic homogeneity estimation of muscle-invasive urinary bladder cancer in an individual prognosis: a mapping study. Urology. 2005; 66(5): 1122-1126, doi: 10.1016/j.urology.2005.06.134, indexed in Pubmed: 16286151.

4. Domanowska E, Jozwicki W, Domaniewski J, et al. Muscle-invasive urothelial cell carcinoma of the human bladder: multidirectional differentiation and ability to metastasize. Hum Pathol. 2007; 38(5): 741-746, doi: 10.1016/j.humpath.2006.11.001, indexed in Pubmed: 17306328

5. Jóźwicki W, Brożyna AA, Siekiera J, et al. Frequency of CD4+CD25+Foxp3+ cells in peripheral blood in relation to urinary bladder cancer malignancy indicators before and after surgical removal. Oncotarget. 2016; 7(10): 11450-11462, doi: 10.18632/oncotarget.7199, indexed in Pubmed: 26862849.

6. Jóźwicki W, Brożyna AA, Siekiera J, et al. Expression of RCAS1 correlates with urothelial bladder cancer malignancy. Int J Mol Sci. 2015; 16(2): 3783-3803, doi: 10.3390/ijms16023783, indexed in Pubmed: 25674852.

7. Jóźwicki W, Brożyna AA, Siekiera J. Expression of OCT4A: the first step to the next stage of urothelial bladder cancer progression. Int J Mol Sci. 2014; 15(9): 16069-16082, doi: 10.3390/ijms150916069, indexed in Pubmed: 25216339.

8. Sharma P, Allison JP. The future of immune checkpoint therapy. Science. 2015; 348(6230): 56-61, doi: 10.1126/science.aaa8172, indexed in Pubmed: 25838373.

9. Okazaki T, Honjo T. PD-1 and PD-1 ligands: from discovery to clinical application. Int Immunol. 2007; 19(7): 813-824, doi: 10.1093/intimm/dxm057, indexed in Pubmed: 17606980.

10. Szabados B, van Dijk N, Tang YZ, et al. Response Rate to Chemotherapy After Immune Checkpoint Inhibition in Metastatic Urothelial Cancer. Eur Urol. 2018; 73(2): 149-152, doi: 10.1016/j.eururo.2017.08.022, indexed in Pubmed: 28917596.

11. Jóźwicki W, Skok Z, Brożyna A, et al. Urological Oncology Prognostic and diagnostic implications of histological differentiation in invasive urothelial cell carcinoma of the bladder: variant or non-classic differentiation number. Central European Journal of Urology. 2010; 63 : 112-116, doi: 10.5173/ceju.2010.03 art1.

12. Jóźwicki W. VENTANA PD-L1 (SP142) - principles of pathomorphological evaluation in urinary bladder cancer. ; 2017.

13. Kim SP, Frank I, Cheville JC, et al. The impact of squamous and glandular differentiation on survival after radical cystectomy for urothelial carcinoma. J Urol. 2012; 188(2): 405-409, doi: 10.1016/j. juro.2012.04.020, indexed in Pubmed: 22704101.

14. Huang $Y$, Zhang SD, McCrudden $C$, et al. The prognostic significance of PD-L1 in bladder cancer. Oncol Rep. 2015; 33(6): 3075-3084, doi: 10.3892/or.2015.3933, indexed in Pubmed: 25963805.

15. Tzeng A, Diaz-Montero CM, Rayman PA, et al. Immunological Correlates of Response to Immune Checkpoint Inhibitors in Metastatic Urothelial Carcinoma. Target Oncol. 2018; 13(5): 599-609, doi: 10.1007/s11523-018-0595-9, indexed in Pubmed: 30267200.
16. Pichler R, Fritz J, Lackner F, et al. Prognostic Value of Testing PD-L1 Expression After Radical Cystectomy in High-risk Patients. Clin Genitourin Cancer. 2018; 16(5): e1015-e1024, doi: 10.1016/j.clgc.2018.05.015, indexed in Pubmed: 29960831.

17. Driver BR, Miller RA, Miller T, et al. Programmed Death Ligand-1 (PDL1) Expression in Either Tumor Cells or Tumor-Infiltrating Immune Cells Correlates With Solid and High-Grade Lung Adenocarcinomas. Arch Pathol Lab Med. 2017; 141(11): 1529-1532, doi: 10.5858/arpa.20170028-OA, indexed in Pubmed: 28829153.

18. El Jabbour T, Ross JS, Sheehan CE, et al. PD-L1 protein expression in tumour cells and immune cells in mismatch repair protein-deficient and -proficient colorectal cancer: the foundation study using the SP142 antibody and whole section immunohistochemistry. J Clin Pathol. 2018; 71(1): 46-51, doi: 10.1136/jclinpath-2017-204525, indexed in Pubmed: 28667193.

19. Faraj SF, Munari E, Guner G, et al. Assessment of tumoral PD-L1 expression and intratumoral CD8 + T cells in urothelial carcinoma. Urology. 2015; 85(3): 703.e1-703.e6, doi: 10.1016/j.urology.2014.10.020 indexed in Pubmed: 25733301.

20. Rosenberg JE, Hoffman-Censits J, Powles T, et al. Atezolizumab in patients with locally advanced and metastatic urothelial carcinoma who have progressed following treatment with platinum-based chemotherapy: a single-arm, multicentre, phase 2 trial. Lancet. 2016; 387(10031): 1909-1920, doi: 10.1016/S0140-6736(16)00561-4, indexed in Pubmed: 26952546.

21. Powles T, Eder JP, Fine GD, et al. MPDL3280A (anti-PD-L1) treatment leads to clinical activity in metastatic bladder cancer. Nature. 2014; 515(7528): 558-562, doi: 10.1038/nature13904, indexed in Pubmed: 25428503

22. Markowitz GJ, Havel LS, Crowley MJp, et al. Immune reprogramming via PD-1 inhibition enhances early-stage lung cancer survival. JCl Insight. 2018; 3(13), doi: 10.1172/jci.insight.96836, indexed in Pubmed: 29997286.

23. Ohaegbulam KC, Assal A, Lazar-Molnar E, et al. Human cancer immunotherapy with antibodies to the PD-1 and PD-L1 pathway. Trends Mol Med. 2015; 21(1): 24-33, doi: 10.1016/j.molmed.2014.10.009, indexed in Pubmed: 25440090.

24. Bellmunt J, de Wit R, Vaughn DJ, et al. KEYNOTE-045 Investigators. Pembrolizumab as Second-Line Therapy for Advanced Urothelial Carcinoma. N Engl J Med. 2017; 376(11): 1015-1026, doi: 10.1056/NEJMoa1613683, indexed in Pubmed: 28212060.

25. Plimack ER, Bellmunt J, Gupta S, et al. Safety and activity of pembrolizumab in patients with locally advanced or metastatic urothelial cancer (KEYNOTE-012): a non-randomised, open-label, phase 1b study. Lancet Oncol. 2017; 18(2): 212-220, doi: 10.1016/S14702045(17)30007-4, indexed in Pubmed: 28081914.

26. Zhang X, Shi X, Li J, et al. PD-1 Blockade Overcomes Adaptive Immune Resistance in Treatment with Anchored-GM-CSF Bladder Cancer Cells Vaccine. J Cancer. 2018; 9(23): 4374-4381, doi: 10.7150/jca.25423, indexed in Pubmed: 30519342.

27. de Jong JJ, Stoop H, Nieboer D, et al. Concordance of PD-L1 expression in matched urothelial bladder cancer specimens. Histopathology. 2018; 73(6): 983-989, doi: 10.1111/his.13710, indexed in Pubmed: 30003574 .

28. He J, Hu Y, Hu M, et al. Development of PD-1/PD-L1 Pathway in Tumor Immune Microenvironment and Treatment for Non-Small Cell Lung Cancer. Sci Rep. 2015; 5: 13110, doi: 10.1038/srep13110, indexed in Pubmed: 26279307. 